\title{
Non-equilibrium Statistical Mechanics of Anharmonic Crystals with Self-consistent Stochastic Reservoirs
}

\author{
Emmanuel Pereira ${ }^{1}$ and Ricardo Falcao ${ }^{2}$ \\ Departamento de Física-ICEx, UFMG, CP 702, 30.161-970 Belo Horizonte MG, Brazil \\ e-mail: ${ }^{1}$ emmanuel@fisica.ufmg.br; ${ }^{2}$ rfalcao@fisica.ufmg.br
}

July 3,2018

\begin{abstract}
We consider a $d$-dimensional crystal with an arbitrary harmonic interaction and an anharmonic on-site potential, with stochastic Langevin heat bath at each site. We develop an integral formalism for the correlation functions that is suitable for the study of their relaxation (time decay) as well as their behavior in space. Furthermore, in a perturbative analysis, for the one-dimensional system with weak coupling between the sites and small quartic anharmonicity, we investigate the steady state and show that the Fourier's law holds. We also obtain an expression for the thermal conductivity (for arbitrary next-neighbor interactions) and give the temperature profile in the steady state.
\end{abstract}

PACS: 05.70.Ln; 05.40.-a; 05.45.-a; 44.10.+i

Short title: Anharmonic Crystals with Self-consistent Reservoirs

\section{Introduction}

We are surrounded by phenomena involving non-equilibrium processes, but our understanding of such systems, i.e., the number of models that permit detailed calculations, is very limited. In particular, it is unknown a simple way of finding the properties in the steady states: e.g., a rigorous derivation of the (phenomenological) Fourier's law from a microscopic anharmonic Hamiltonian model has not been established up to now (see [1, 2] for a review). It makes the analysis of simple dynamical models describing non-equilibrium processes a problem of interest.

A commonly studied microscopic model is the Hamiltonian chain (or its d-dimensional version) of $\mathrm{N}$ interacting oscillators coupled to heat baths at each site or at the boundaries only, and its anharmonic version with small quartic on-site interactions.

For the harmonic case of the model with thermal reservoirs at the boundaries, the covariance of the stationary state has been calculated in [3], long time ago. There, it is shown that the heat current is independent on the length of the chain, and so, the Fourier's law does not hold. The temperature profile is also computed in [3]: the temperature is essencially constant in the interior of the chain, but decreases exponentially close to the hotter bath and increases close to the opposite end. I.e., the profile has the lowest temperature near the hottest reservoir and the highest temperature near the coldest reservoir. For the anharmonic case, there are interesting and recent results. The existence of steady states is proved in [4, and the positivity of entropy 
production in [5]. Numerical results strongly suggest that the Fourier's law holds in such a case [6], 7], but, in the opposite direction, a perturbative analysis [8] shows that the heat current does not depend on the size of the system. Also in the perturbative study $[\underline{8}$, as in harmonic case [3], the temperature profile (discarding the exponential decay in the bulk of the chain) is in the "wrong" way: the hottest temperature is near the coldest bath, and vice-versa. In short, it is unclear whether the Fourier's law holds or not in such anharmonic models. It is worth to recall that other results also indicate that it is wrong the opinion that the sole anharmonicity of the on-site potential shall ensure normal heat conductivity in some commonly used models [9].

The harmonic crystal model with next-neighbor interactions and heat bath at each site has been recently analyzed in [10]. It is proved, for a uniquely fixed temperature profile leading to the steady state (given the temperatures at the boundaries), that the heat current satisfies the Fourier's law. For the case of more intricate interactions (intense and beyond next-neighbor sites), for a chain with some few sites, some results presented in [11] indicate that there is a "strange" heat flux in the harmonic network (and the authors claim that the results persist under weak anharmonic perturbations): inside the chain, the direction of the heat fluxes can not (in general) be supposed from the temperature of the heat baths.

In the present paper, also with the aim of studying the dynamics of simple microscopic models in order to understand properties of non-equilibrium systems, we study the anharmonic version of this crystal with stochastic Langevin heat bath at each site (model named as crystal with selfconsistent reservoirs). We describe an approach and obtain an integral formalism suitable for the study of the correlation functions (of the d-dimensional system with quite general interactions). Furthermore, using perturbative calculations, for a weak coupling between the sites and a weak anharmonic potential, we show (for the one-dimensional system) that the Fourier's law still holds. That is, we show (at least up to first order in the perturbative computation) that the Fourier's law is valid for this microscopic anharmonic Hamiltonian model. We also obtain an expression for the thermal conductivity (for next-neighbor interactions which may arbitrarily change along the chain), and give the temperature profile in the steady state. For the simpler case of nextneighbor interactions constant along the chain, our results (considering the anharmonic model) coincide with those of the harmonic case recently described in [10].

The rest of the paper is organized as follows. In section 2 we present the model and some expressions for the energy current. The integral formalism for the correlation functions is developed in section 3 . In section 4 , in a perturbative computation, we analyze the energy current in the steady state and the Fourier's law. In section 5 we argue on the reliability of the perturbative results and present some concluding remarks.

\section{The Model and Initial Considerations}

Lets us introduce the model to be analyzed here and some expressions for the energy current. We consider the stochastic Langevin dynamics of an anharmonic crystal, i.e. a scalar field lattice model with unbounded spin variables in a d-dimensional lattice space box $\Lambda \subset \mathbb{Z}^{d}$, with stochastic heat bath at each site. Precisely, we take a system of $N$ oscillators with Hamiltonian

$$
H(q, p)=\sum_{j=1}^{N} \frac{1}{2}\left[p_{j}^{2}+M q_{j}^{2}\right]+\frac{1}{2} \sum_{j \neq l=1}^{N} q_{l} J_{l j} q_{j}+\sum_{j=1}^{N} \lambda \mathcal{P}\left(q_{j}\right)
$$


where $M>0, \mathcal{P}$ gives the anharmonic on-site perturbation (e.g., $\mathcal{P}\left(q_{j}\right)=q_{j}^{4}$ ), and we consider the time evolution given by the stochastic differential equations

$$
\begin{aligned}
d q_{j} & =p_{j} d t, \quad j=1, \ldots, N, \\
d p_{j} & =-\frac{\partial H}{\partial q_{j}} d t-\zeta p_{j} d t+\gamma_{j}^{1 / 2} d B_{j}, \quad j=1, \ldots, N,
\end{aligned}
$$

where $B_{j}$ are independent Wiener processes, i.e., $d B_{j} / d t$ are independent white noises; $\zeta$ is the heat bath coupling; and $\gamma_{j}=2 \zeta T_{j}$, where $T_{j}$ is the temperature of the $\mathrm{j}$-th heat bath.

To describe the energy current in the system, we write the local energy of the spin (oscillator) $j$ as

$$
H_{j}(q, p)=\frac{1}{2} p_{j}^{2}+U^{(1)}\left(q_{j}\right)+\frac{1}{2} \sum_{l \neq j} U^{(2)}\left(q_{j}-q_{l}\right),
$$

where the expression for $U^{(1)}$ and $U^{(2)}$ follow immediately from (11) and $\sum_{j=1}^{N} H_{j}=H$. Then, we have

$$
\left\langle\frac{d H_{j}(t)}{d t}\right\rangle=\left\langle R_{j}(t)\right\rangle-\left\langle\mathcal{F}_{j<}-\mathcal{F}_{j>}\right\rangle
$$

where $\langle\cdot\rangle$ denotes the expectation with respect to the noise distribution, and

$$
\left\langle R_{j}(t)\right\rangle=\zeta\left(T_{j}-\left\langle p_{j}^{2}\right\rangle\right)
$$

gives the energy flux from the $\mathrm{j}$-th reservoir to the $\mathrm{j}$-th site. The energy current inside the system is given by $\mathcal{F}_{j}$, where

$$
\begin{aligned}
& \mathcal{F}_{j<}=\sum_{l>j} \nabla U^{(2)}\left(q_{j}-q_{l}\right) \frac{p_{j}+p_{l}}{2} \\
& \mathcal{F}_{j>}=\sum_{l<j} \nabla U^{(2)}\left(q_{l}-q_{j}\right) \frac{p_{l}+p_{j}}{2} .
\end{aligned}
$$

In particular, in the steady state we have $\left\langle d H_{j}(t) / d t\right\rangle=0$. We will turn to these expressions to discuss the Fourier's law later.

\section{The Integral Formalism for the Correlation Functions}

For convenience, we introduce the phase-space vector $\phi=(q, p)$ with $2 N$ coordinates and write the equation for the dynamics (2) as

$$
\dot{\phi}=-A \phi-\lambda \mathcal{P}^{\prime}(\phi)+\sigma \eta
$$

where $A=\left(A^{0}+\mathcal{J}\right)$ and $\sigma$ are $2 N \times 2 N$ matrices given by

$$
A^{0}=\left(\begin{array}{cc}
0 & -I \\
\mathcal{M} & \Gamma
\end{array}\right), \quad \mathcal{J}=\left(\begin{array}{ll}
0 & 0 \\
J & 0
\end{array}\right), \quad \sigma=\left(\begin{array}{cc}
0 & 0 \\
0 & \sqrt{2 \Gamma \mathcal{T}}
\end{array}\right)
$$

$I$ above is the unit $N \times N$ matrix; $J$ is the $N \times N$ matrix for the two site interaction $J_{l j}$ (see(11)); and $\mathcal{M}, \Gamma, \mathcal{T}$ are diagonal $N \times N$ matrices: $\mathcal{M}_{j l}=M \delta_{j l}, \Gamma_{j l}=\zeta \delta_{j l}, \mathcal{T}_{j l}=T_{j} \delta_{j l} . \eta$ are independent white-noises; $\mathcal{P}^{\prime}(\phi)$ is a $2 N \times 1$ matrix with $\mathcal{P}^{\prime}(\phi)_{j}=0$ for $j=1, \ldots, N$ and

$$
\mathcal{P}^{\prime}(\phi)_{i}=\frac{d \mathcal{P}\left(\phi_{i-N}\right)}{d \phi_{i-N}} \quad \text { for } \quad i=N+1, \ldots, 2 N .
$$


To describe the dynamics we first consider the system without the coupling $J$ among the sites and without the anharmonic perturbation $(\lambda=0)$ (interactions which we include in a second step). Then the (straightforward) solution of (7) above with $J \equiv 0, \lambda=0$ is the Ornstein-Uhlenbeck process given by

$$
\phi(t)=e^{-t A^{0}} \phi(0)+\int_{0}^{t} d s e^{-(t-s) A^{0}} \sigma \eta(s) .
$$

For simplicity we take $\phi(0)=0$. The covariance of this Gaussian process evolves as

$$
\begin{aligned}
\langle\phi(t) \phi(s)\rangle_{0} \equiv \mathcal{C}(t, s) & = \begin{cases}e^{-(t-s) A^{0}} \mathcal{C}(s, s) & t \geq s, \\
\mathcal{C}(t, t) e^{-(s-t) A^{0^{T}}} & t \leq s,\end{cases} \\
\mathcal{C}(t, t) & =\int_{0}^{t} d s e^{-s A^{0}} \sigma e^{-s A^{0^{T}}} .
\end{aligned}
$$

It is easy to see (e.g. diagonalizing $A^{0}$ ) that

$$
\exp \left(-t A^{0}\right)=e^{-t \frac{\zeta}{2}} \cosh (t \rho)\left\{I+\frac{\tanh (t \rho)}{\rho}\left(\begin{array}{cc}
\frac{\zeta}{2} I & -I \\
-\mathcal{M} & -\frac{\zeta}{2} I
\end{array}\right)\right\}
$$

(and a similar expression follows for the transpose $\exp \left(-t A^{0^{T}}\right)$ ), where $I$ is the $N \times N$ unit matrix, etc; $\rho=\left((\zeta / 2)^{2}-M\right)^{1 / 2}$ (we assume that $(\zeta / 2)^{2}>M>0$ ). In this simple case (of $J \equiv 0, \lambda=0$ ), as $t \rightarrow \infty$ we have a convergence to equilibrium (any single site is isolate) and the stationary state is Gaussian, with mean zero and covariance

$$
C=\int_{0}^{\infty} d s \quad e^{-s A^{0}} \sigma e^{-s A^{0^{T}}}=\left(\begin{array}{cc}
\frac{\mathcal{T}}{M} & 0 \\
0 & \mathcal{T}
\end{array}\right),
$$

where, again, $\mathcal{T}$ is a diagonal matrix with elements $T_{i} \delta_{i j}$ (in short, for any site we have a Gibbs measure at temperature $T_{i}$ ).

To introduce the coupling interactions and the anharmonic potential, we use a tool of general theory of stochastic differential equations, namely the Girsanov theorem [12. It gives a measure $\rho$ for the new process (7) as a "perturbation" of the measure $\mu_{\mathcal{C}}$ associated to the process with $J \equiv 0, \lambda=0$. Precisely, for any measurable set $A$, it states that $\rho(A)=E_{0}\left(1_{A} Z(t)\right)$, where $E_{0}$ is the expectation for $\mu_{\mathcal{C}}$ (the process with $J \equiv 0, \lambda=0$ ); $1_{A}$ denotes the characteristic function, and

$$
\begin{aligned}
Z(t) & =\exp \left(\int_{0}^{t} u \cdot d B-\frac{1}{2} \int_{0}^{t} u^{2} d s\right), \\
\gamma_{i}^{1 / 2} u_{i} & =-\mathcal{J}_{i k} \phi_{k}-\lambda \mathcal{P}^{\prime}(\phi)_{i},
\end{aligned}
$$

(the inner products above are in $\mathbb{R}^{2 N}$ ). From (8) and the expression above for $u_{i}$, note that $u_{i}$ is nonvanishing only for $i>N$ (i.e., $i \in[N+1, N+2, \ldots, 2 N]$ ). In what follows we will use the following index notation: $i$ for index values in the set $[N+1, N+2, \ldots, 2 N], j$ for values in the set $[1,2, \ldots, N]$, and $k$ for values in $[1,2, \ldots, 2 N]$.

For clearness, let us rewrite the stochastic equations for the initial process (with $J \equiv 0$, $\lambda=0)$ as

$$
\begin{aligned}
d \phi_{j} & =-A_{j k}^{0} \phi_{k} d t, \quad j \in[1, \ldots, N] \\
d \phi_{i} & =-A_{i k}^{0} \phi_{k} d t+\gamma_{i}^{1 / 2} d B_{i}, \quad i \in[N+1, \ldots, 2 N],
\end{aligned}
$$


the sum over $k$ (in $[1,2 \ldots, 2 N]$ ) is assumed above (as well as obvious sum over some indices in what follows).

Turning to the terms in $Z(t)$ we have

$$
\begin{aligned}
u_{i} d B_{i}=\gamma_{i}^{-1 / 2} u_{i} \cdot \gamma_{i}^{1 / 2} d B_{i} & =\gamma_{i}^{-1 / 2} u_{i} \cdot\left(d \phi_{i}+A_{i k}^{0} \phi_{k} d t\right) \\
& =\left(-\gamma_{i}^{-1} \mathcal{J}_{i j} \phi_{j}-\gamma_{i}^{-1} \lambda \mathcal{P}^{\prime}(\phi)_{i}\right)\left(d \phi_{i}+A_{i k}^{0} \phi_{k} d t\right)
\end{aligned}
$$

that follows from (15) and (16) above. We still use Itô formula to write the terms with $d \phi_{i}$ as

$$
\begin{aligned}
-\gamma_{i}^{-1} \mathcal{J}_{i j} \phi_{j} d \phi_{i} & =-d F_{1}-\gamma_{i}^{-1} \phi_{i} \mathcal{J}_{i j} A_{j k}^{0} \phi_{k} d t, \\
F_{1}(\phi) & =\gamma_{k}^{-1} \phi_{i} \mathcal{J}_{i j} \phi_{j} .
\end{aligned}
$$

With similar manipulations we obtain

$$
\begin{aligned}
Z(t) \equiv & \exp \left(\int_{0}^{t} u \cdot d B-\frac{1}{2} \int_{0}^{t} u^{2} d s\right) \\
= & \exp \left\{-F_{1}(\phi(t))+F_{1}(\phi(0))-\lambda F_{2}(\phi(t))+\lambda F_{2}(\phi(0))\right\} \\
& \times \exp \left\{-\int_{0}^{t} W_{J}(\phi(s)) d s-\int_{0}^{t} \lambda W_{\lambda}(\phi(s)) d s-\int_{0}^{t} \lambda W_{\lambda J}(\phi(s)) d s\right\},
\end{aligned}
$$

with

$$
\begin{aligned}
F_{1}(\phi(t))= & \gamma_{i}^{-1} \phi_{i}(t) \mathcal{J}_{i j} \phi_{j}(t), \quad F_{2}(\phi(t))=\gamma_{i}^{-1} \mathcal{P}^{\prime}(\phi)_{i}(t) \phi_{i}(t) \\
W_{J}(\phi(s))= & \gamma_{i}^{-1} \phi_{i}(s) \mathcal{J}_{i j} A_{j k}^{0} \phi_{k}(s)+\phi_{k}(s) A_{k i}^{0^{T}} \gamma_{i}^{-1} \mathcal{J}_{i j} \phi_{j}(s)+ \\
& +\frac{1}{2} \phi_{j^{\prime}}(s) \mathcal{J}_{j^{\prime} i}^{T} \gamma_{i}^{-1} \mathcal{J}_{i j} \phi_{j}(s), \\
\lambda W_{\lambda}(\phi(s))= & \lambda \gamma_{i}^{-1} \phi_{i}(s) \mathcal{P}^{\prime \prime}(\phi)_{i}(s) A_{i-N, k}^{0} \phi_{k}(s)+\lambda \gamma_{i}^{-1} \mathcal{P}^{\prime}(\phi)_{i}(s) A_{i k}^{0} \phi_{k}(s)+ \\
& +\frac{1}{2} \lambda^{2} \gamma_{i}^{-1}\left(\mathcal{P}^{\prime}(\phi)_{i}\right)^{2}(s) A_{i-N, k}^{0} \phi_{k}(s) . \\
\lambda W_{\lambda J}(\phi(s))= & \lambda \gamma_{i}^{-1} \mathcal{P}^{\prime}(\phi)_{i}(s) \mathcal{J}_{i j} \phi_{j}(s) .
\end{aligned}
$$

And so, for the expectations, considering the process with coupling between sites and anharmonic perturbation, we have, e.g. for the two-point function,

$$
\left\langle\phi_{u}\left(t_{1}\right) \phi_{v}\left(t_{2}\right)\right\rangle=\int \phi_{u}\left(t_{1}\right) \phi_{v}\left(t_{2}\right) Z(t) d \mu_{\mathcal{C}}(\phi), \quad t_{1}, t_{2}<t .
$$

The formula above, a Feynman-Kac type integral representation, is suitable for the study of general n-point correlation functions: for the analysis of their time decay (relaxation properties), space behavior, etc. In particular, we will analyze the energy current in the steady state, problem that involves the investigation of terms such as $\lim _{t \rightarrow \infty}\left\langle\phi_{i}(t) \phi_{j}(t)\right\rangle$, see (6) .

\section{The Heat Flow and Fourier's Law}

To study the heat flow in the steady state we need to analyze the two-point correlation functions given by formula (6). The averages over the stationary distributions will be obtained as the limit

$$
\left\langle\phi_{u} \phi_{v}\right\rangle=\lim _{t \rightarrow \infty}\left\langle\phi_{u}(t) \phi_{v}(t)\right\rangle=\lim _{t \rightarrow \infty} \int \phi_{u}(t) \phi_{v}(t) Z(t) d \mu_{\mathcal{C}}(\phi) .
$$


We will establish conditions for the convergence to the steady state later.

To carry out the computation, note that $\mathcal{C}(t, s)$, given by (11)-(14), may be written as (for $t>s)$

$$
\mathcal{C}(t, s)=\exp \left(-(t-s) A^{0}\right) C+\mathcal{O}(\exp [-(t+s) \zeta / 2])
$$

and the effects of the second term in the r.h.s of the equation above disappears in the correlation formula in the limit of $t \rightarrow \infty$.

For the anharmonic interaction we choose, for ease of computation, $\mathcal{P}(\phi)_{i}(s)=\frac{a_{4}}{4}: \phi_{i-N}^{4}(s)$ :, where the dots mean the Wick order with respect to the Gaussian measure $\mu_{\mathcal{C}}$.

We will make a perturbative analysis, i.e., we will assume that the coupling between two sites $J$ as well as the anharmonic potential coefficient $\lambda$ are small. Hence, up to first order in $J$ and $\lambda$, after (considerable but straightforward) calculations we have

$$
\begin{aligned}
& \left\langle\phi_{u} \phi_{v}\right\rangle= \\
& \left\{\begin{array}{l}
\frac{1}{2 \zeta M}\left[\mathcal{J}_{v+N, u-N} T_{u-N}-\mathcal{J}_{u, v} T_{v}\right] \delta_{u-N, v} \\
T_{u} \delta_{u, v} \quad \text { for } u, v \in[N+1, \ldots, 2 N] .
\end{array} \text { for } u \in[N+1, \ldots, 2 N], v \in[1, \ldots, N],\right.
\end{aligned}
$$

For simplicity we will restrict the analysis of the energy current to one-dimensional systems only. From (6) we have

$$
\mathcal{F}_{j<}=\sum_{\substack{r>j \\ r \in[1, \ldots, N]}} \mathcal{J}_{j+N, r}\left(\phi_{j}-\phi_{r}\right) \frac{\left(\phi_{j+N}+\phi_{r+N}\right)}{2},
$$

where $\left\langle\mathcal{F}_{j<}\right\rangle$ denotes de energy flow between site $j$ and the site $r$ (with $r>j$ ) connected by the interaction $\mathcal{J}$. Using the results describe in (19) above we obtain

$$
\left\langle\mathcal{F}_{j<}\right\rangle=\sum_{r>j} \frac{\left(\mathcal{J}_{j+N, r}\right)^{2}}{2 \zeta M}\left(T_{r}-T_{j}\right) .
$$

Let us analyze, in particular, the case of next-neighbor interaction only. In such a case

$$
\mathcal{F}_{j \rightarrow j+1} \equiv\left\langle\mathcal{F}_{j<}\right\rangle=\frac{\mathcal{J}_{j+N, j+1}}{2 \zeta M}\left(T_{j+1}-T_{j}\right)
$$

The condition $\left\langle d H_{i} / d t\right\rangle=0$, that characterizes the stationary state , together with expressions (415) and $\left\langle R_{j}(t)\right\rangle=0$ (that comes from (5) and (19)) lead to

$$
\mathcal{F}_{1 \rightarrow 2}=\mathcal{F}_{2 \rightarrow 3}=\mathcal{F}_{3 \rightarrow 4}=\ldots=\mathcal{F}_{N-1 \rightarrow N} .
$$

I.e., using the notation $J_{j} \equiv\left(\mathcal{J}_{j+N, j+1}\right)^{2} / 2 \zeta M$,

$$
J_{1}\left(T_{2}-T_{1}\right)=J_{2}\left(T_{3}-T_{2}\right)=J_{3}\left(T_{4}-T_{3}\right)=\ldots=J_{N-1}\left(T_{N}-T_{N-1}\right) .
$$

It is easy to see that given the temperatures at the boundaries $T_{1}$ and $T_{N}$, and nonvanishing $J_{1}, J_{2}, \ldots, J_{N-1}$, there exists an unique solution $T_{2}, T_{3}, \ldots, T_{N-1}$ for the linear system of equations above (24). Namely, we obtain

$$
T_{k}=T_{1}+\left(\frac{1}{J_{1}}+\frac{1}{J_{2}}+\ldots+\frac{1}{J_{N-1}}\right)^{-1} \times\left(\frac{1}{J_{1}}+\frac{1}{J_{2}}+\ldots+\frac{1}{J_{k-1}}\right)\left(T_{N}-T_{1}\right),
$$


that determines the temperature profile in the steady state. Note that it is a monotonic function, oriented in the "right" way: the hottest temperature is near the hottest bath, and vice-versa.

For the energy current we get

$$
J_{1}\left(T_{2}-T_{1}\right)=\ldots=J_{j}\left(T_{j+1}-T_{j}\right)=\chi \frac{\left(T_{N}-T_{1}\right)}{N-1}, \quad \frac{\chi}{N-1}=\left(\frac{1}{J_{1}}+\frac{1}{J_{2}}+\ldots+\frac{1}{J_{N-1}}\right)^{-1},
$$

that is, the Fourier's law still holds. For the simpler case of the same interaction between two any next-neighbor sites, i.e. $J_{1}=J_{2}=\ldots=J_{N-1}$, we have, for the thermal conductivity,

$$
\chi=J_{1}=\frac{\left(\mathcal{J}_{1+N, 2}\right)^{2}}{2 \zeta M} .
$$

For comparison, in [10] the authors treat the linear dynamical problem, i.e. (7) with $\lambda=0$ and

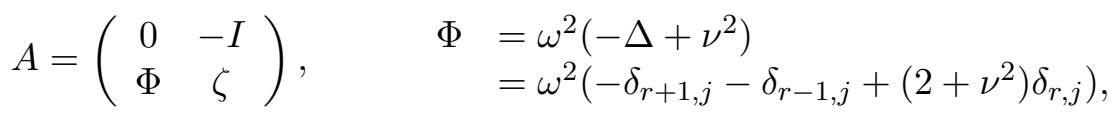

and obtain (in a nonperturbative approach)

$$
\chi=\frac{\omega^{2}}{\zeta} \frac{1}{\left[2+\nu^{2}+\sqrt{\nu^{2}\left(4+\nu^{2}\right)}\right]} .
$$

In our case (considering the same $J$ of [10]), we have

$$
A=\left(\begin{array}{cc}
0 & -I \\
J+\mathcal{M} & \zeta
\end{array}\right), \quad \begin{aligned}
& J=\omega^{2}\left(-\delta_{r+1, j}-\delta_{r-1, j}\right) \\
& \mathcal{M}=M \delta_{r j}, \quad M=\omega^{2}\left(2+\nu^{2}\right)
\end{aligned}
$$

and so, our formula (27) above becomes

$$
\chi=\frac{\left(-\omega^{2}\right)^{2}}{2 \zeta\left(2+\nu^{2}\right) \omega^{2}}=\frac{\omega^{2}}{\zeta\left(4+2 \nu^{2}\right)} .
$$

Considering that our computation was carried out in a perturbative approach with small $J$ but $M$ not small (see the comments at the final section ), i.e., $\omega^{2}$ small, $\nu$ large, we have in (29)

$$
\sqrt{\nu^{2}\left(4+\nu^{2}\right)} \approx \nu^{2}\left(1+\frac{1}{2} \frac{4}{\nu^{2}}\right)=\nu^{2}+2
$$

That is, our computation, when restricted to the case treated in [10, leads to the same result.

In short, we have shown (in a perturbative analysis: up to first order in $\lambda$ and $J$ ) that the Fourier's law still holds for the harmonic crystal with self-consistent reservoirs when a small nonharmonic on site perturbation is introduced in the interaction.

\section{Concluding Remarks}

The approach presented here establishes an integral representation for the correlation functions, say, a Feynman-Kac type formalism. That is, in some sense, we map the stochastic problem on a non-canonical field theory. Such an approach is inspired on previous works considering the 
study of the relaxation to equilibrium of some nonconservative stochastic Langevin systems [13, [14, 15], 16, 17. There, the time decay of the two and four-point functions is analyzed in detail. A perturbative study is carried out within the integral formalism in the regimes of low and high temperature. In the low temperature region, for the system with a weak anharmonic potential and a bare mass (the coefficient of the local quadratic term) large enough, it is proved that the perturbative analysis is not naive: e.g., the rigorous results described in [14] show that the complete treatment of the four-point function adds only small corrections to the behavior obtained by the perturbative calculations presented in [13. Using similar techniques (cluster expansions, etc) we expect to prove the results about the behavior of the correlations presented here (for small $\lambda$, nonzero $M$ and $\zeta$, and $T_{j}$ not large). The perturbative analysis of our system with all the reservoirs at (different but) high temperature (i.e., with the perturbative parameter given by $1 / T_{j}$ instead of $\lambda$ ) shall be possible following procedures similar to those described in [16] and references there in.

Another interesting open problem is the behavior of the system in the limit of the coupling with the interior heat bath taken to zero: in such a case, as we have mentioned before (compare [6] and [7] with [8]), it is not clear if the Fourier's law is valid or not.

Acknowlegment. Work partially supported by CNPq and CAPES (Brazil).

\section{References}

[1] F. Bonetto, J. L. Lebowitz and L. Rey-Bellet, in Mathematical Physics 2000, edited by A. Fokas, A. Grigoryan, T. Kibble, and B. Zegarlinski (Imperial College, London, 2000), p. 128.

[2] S. Lepri, R. Livi and A. Politi, Phys. Rep. 377, 80 (2003).

[3] Z. Rieder, J. L. Lebowtiz and E. Lieb, J. Math. Phys. 8, 1073 (1967).

[4] J. P. Eckmann, C. A. Pillet and L. Rey-Bellet, Commun. Math. Phys. 201, 657 (1999).

[5] J. P. Eckmann, C. A. Pillet and L. Rey-Bellet, J. Stat. Phys. 95, 305 (1999).

[6] B. Hu, B. Li and H. Zhao, Phys. Rev.E 57, 2992 (1998).

[7] K. Aoki and D. Kusnezov, Ann. Phys. 295, 50 (2002).

[8] R. Lefereve and A. Schenkel, J. Stat. Phys 115, 1389 (2004).

[9] A.V. Sauin and O.V. Gendelman, Phys. Rev. E 67, 041205 (2003).

[10] F. Bonetto, J. L. Lebowitz and J. Lukkarinen, J. Stat.Phys 116, (2004).

[11] J. P. Eckmann and E. Zabey, J. Stat. Phys. 114, 515 (2004).

[12] B. Øksendal, Stochastic Differential Equations: An Introduction with Applications (Springer-Verlag, Berlin, 1991); I. Karatzas and S. E. Shreve, Brownian Motion and Stochastic Calculus (Springer-Verlag, Berlin, 1991).

[13] R. Schor, J. C. A. Barata, P. A. Faria da Veiga and E. Pereira, Phys. Rev. E 59, 2689 (1999). 
[14] P. A. Faria da Veiga, M. O'Carroll, E. Pereira and R. Schor, Commun. Math. Phys. 220, 377 (2001).

[15] E. Pereira, Phys. Lett. A 282, 169 (2001).

[16] E. Pereira, Phys. Rev. E 65, 056605 (2002).

[17] E. Pereira, Physica D 192, 23 (2004). 